\title{
Nanodiamond aqueous dispersions as potential nanofluids: the determination of properties by thermal lensing and other techniques
}

\author{
L. O. Usoltseva, D. S. Volkov, N. V. Avramenko, M. V. Korobov, M. A. Proskurnin \\ Lomonosov Moscow State University, 119991 Moscow, Russia \\ usoltsevalilya@gmail.com, proskurnin@gmail.com
}

DOI 10.17586/2220-8054-2018-9-1-17-20

\begin{abstract}
Thermal-lens spectrometry was used to characterize thermal diffusivity and thermal conductivity of aqueous nanodiamond dispersions at the level of $\mathrm{mg} / \mathrm{mL}$, accompanied by heat capacity, density, and viscosity measurements and modelling. The data from thermal lensing corresponding to thermal equilibrium show $3-7 \%$ increase in thermal conductivity of the studied dispersions, show good precision and agree with the existing data.
\end{abstract}

Keywords: nanodiamond nanofluid, thermal lensing.

Received: 19 June 2017

Revised: 17 September 2017

\section{Introduction}

More effective cooling fluids are required for nuclear energy industry, operation of solar panels, state-of-the-art computers etc. Recently, nanofluids have been actively studied: they are two-component systems consisting of a base fluid (water or a mineral oil) and dispersed nanoparticles enhancing thermal conductivity. As the latter, metal or oxide nanoparticles are often used. However, carbon nanofluids are more stable, chemically inert, and more environmentally benign. The thermal conductivity of such systems, to date, has not been fully studied and novel experimental techniques are required. Photothermal techniques and thermal-lens spectrometry (TLS) are among the methods widely used for the evaluation of properties of different materials giving both thermal and optical data [1,2]. The objective of this study is thermophysical characterization of aqueous nanodiamond (ND) dispersions. We applied TLS to obtain thermal diffusivity, which was used in calculating thermal conductivity. The specific heat capacities and densities of ND dispersions necessary for such calculations were also measured. The characterization of ND dispersions was implemented with the viscosity determination.

\section{Materials and methods}

\subsection{Photothermal spectrometer and other instruments}

Back-synchronized dual-beam mode-mismatched thermal-lens measurements are described elsewhere [2]. The principle is based on recording an excitation-laser induced (Innova 90-6, Coherent, USA; 488.0 and $514.5 \mathrm{~nm}$; waist diameter, $59.8 \pm 0.5 \mu \mathrm{m}$; power, $1-250 \mathrm{~mW}$ ) refractive heterogeneity (the thermal lens effect) causing defocusing of a collinear He-Ne laser probe beam (SP-106-1, Spectra Physics, USA; $632.8 \mathrm{~nm}$; diameter, $25.0 \pm 0.2 \mu \mathrm{m}$; power, $4 \mathrm{~mW}$ ) and hence a reduction in the probe beam intensity at its center was detected by a far-field photodiode (sample-to-detector distance $120 \pm 0.1 \mathrm{~cm}$ ) supplied with a KS-11 stained-glass bandpass filter and a 2-mm-diameter pinhole and connected to the custom-made software.

DCS-30 TA Mettler instrument (Switzerland) was used to measure specific heats from 288 to $323 \mathrm{~K}$ (scanning rate, $10 \mathrm{~K} / \mathrm{min}$; sample mass, $20-30 \mathrm{mg}$ ). A Kern 770 analytical balance (Germany) was used for dry powder sample weighing. An Ecros 6500 shaker (Russia) and a GRAD 28-35 ultrasound bath from Grad-Technology (Russia) were used for preparing ND dispersions in water. A SNOL 20/300 heating oven (Snol-Term Ltd., Russia) was used for the evaporation of ND aqueous dispersions. A microVISC microfluidic slit rheometer with a microfluidic device, microVISC Type A chipset (13HA05100058, Rheosense Inc., USA) was used to measure steady shear viscosities of ND solutions. Water was used before each measurement as a reference liquid to ensure the accuracy of the rheometer. Densities were determined with a VIP-2MP (Russia) vibrating-tube densimeter. Calibration was performed at $298.15 \mathrm{~K}$ by known densities and oscillation periods of ambient air, ultrapure water and REP-12 standard material $\left(1090.32 \mathrm{~kg} \cdot \mathrm{m}^{-3}\right.$, produced and certified by Mendeleev Institute for Metrology, Russia). The temperature was maintained by the built-in thermostat; the temperature uncertainty was $0.02{ }^{\circ} \mathrm{C}$. The standard deviation for measurements of solution density is $0.1 \mathrm{~kg} \cdot \mathrm{m}^{-3}$. 


\subsection{Materials}

All the reagents and solvents used were of $\mathrm{cp}$ grade or higher. The glassware was washed with acetone followed by conc. nitric acid. Water from a Milli Q water purification system (Millipore, France) was used: pH 6.8, specific resistance $18.2 \mathrm{M} \Omega \mathrm{cm}$. Commercially available NDs (RUDDM and RDDM, powdered "RealDzerzhinsk" Ltd., Dzerzhinsk, Russia and SDND, an aqueous dispersion, PlasmaChem GmbH, Germany) were used throughout. Aqueous dispersions were produced as reported elsewhere [3]. The concentrations of the stock solutions were established by gravimetry. If a stock solution was stored for more than a day, it was manually stirred vigorously. Then, series of working solutions with the concentrations $1-190 \mathrm{mg} / \mathrm{mL}$ for RUDDM, 0.7 $30 \mathrm{mg} / \mathrm{mL}$ for RDDM, and $1-50 \mathrm{mg} / \mathrm{ml}$ for SDND were prepared.

\section{Results and discussion}

Three types of ND capable of forming concentrated solutions were selected for the study. The viscosities of aqueous dispersions are shown in Fig. 1 (left). Concentration dependences are different for the three systems studied, but, as for RUDDM and RDDM, they are similar to the corresponding results of Vul' and collaborators [4]. When the concentration exceeds $50 \mathrm{mg} / \mathrm{ml}$ for RUDDM and $90 \mathrm{mg} / \mathrm{ml}$ for RDDM, the viscosity $v s$. concentration dependence begins to deviate from linearity.
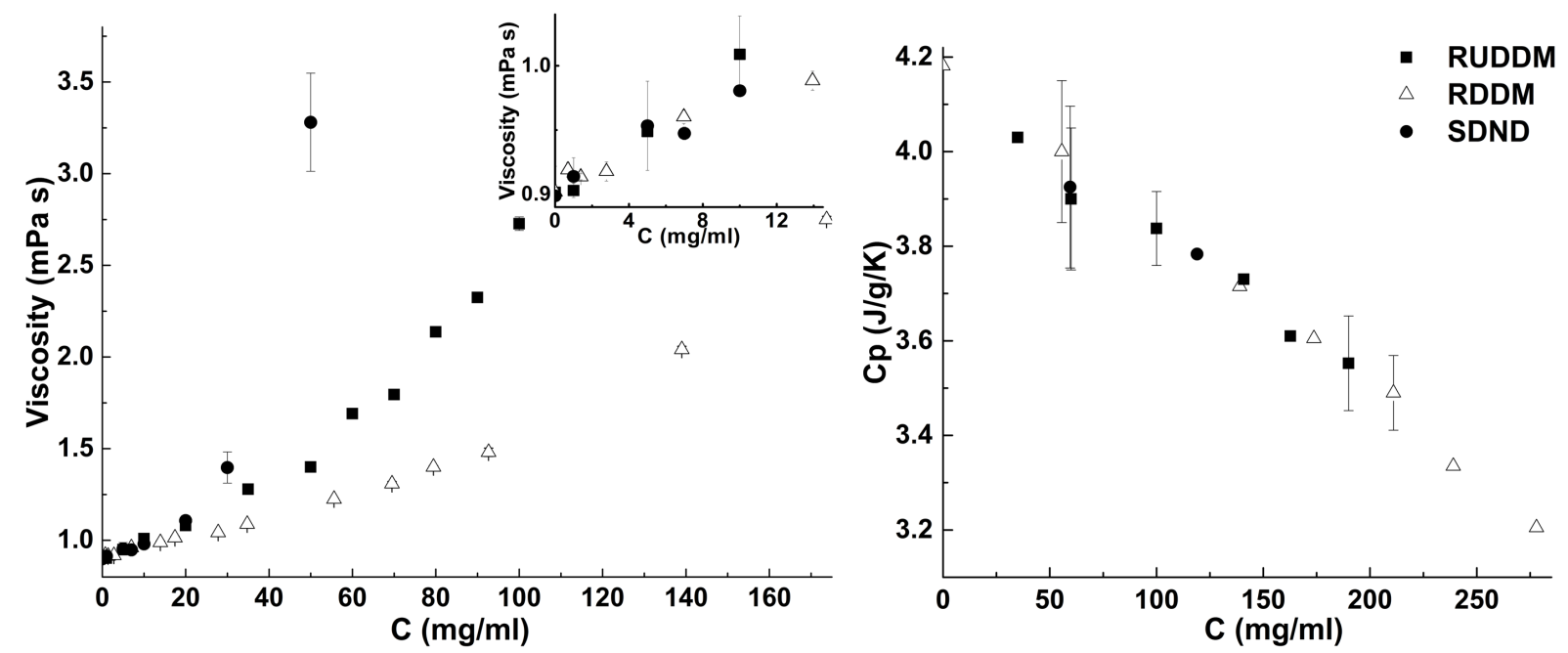

FIG. 1. Viscosity (left) and specific heat (right) of aqueous RUDDM, RDDM and SDND (squares, triangles and circles, respectively) at $25{ }^{\circ} \mathrm{C}$ as functions of concentration. Inset: a larger scale for viscosity at low concentrations

According to Sundar and colleagues [5], available theoretical formulas for viscosity estimation include particle volume concentration to the different power. Since viscosity plays a key role in systems involved in a fluid flow, these dependences should be useful in selecting ND suspensions with the best thermal performance.

The results of density measurements of ND-dispersions are given in Table 1. We observed a linear density $v s$. concentration of ND $(\mathrm{mg} / \mathrm{ml})$ dependences for all the three systems studied. The corresponding slopes and $R^{2}$-factors are given in Table 1 . For $\mu$ m-sized particles, the equation is known for the concentration dependence of density in the two-phase mixtures [6]. Pak and Cho [7] implemented the same equation for nano-sized particles, which is expressed as $\rho_{n f l}=\phi \rho_{p}+(1-\phi) \rho_{f l}$, where $\rho_{n f l}, \rho_{p}, \rho_{f l}$ are densities of the two-phase mixture, particles, and pure fluid, respectively, and $\phi$ is the particle volume fraction. Density measurements of nanofluids of inorganic particles $\left(\mathrm{Al}_{2} \mathrm{O}_{3}, \mathrm{Sb}_{2} \mathrm{O}_{5}, \mathrm{SnO}_{2}\right.$, and $\left.\mathrm{ZnO}\right)$ in 60:40 ethylene glycol/water showed good agreement with this theoretical equation, but the deviation was higher for $\mathrm{ZnO}$ and increased with the particle volume concentration [8]. This equation is used for nanofluid densities for aqueous [9,10], mixed [11], and organic [12] ND dispersions. To use the equation, densities of the base fluid and particles are needed. The latter is determined by a hydrostatic weighing in distilled water [13] or by pycnometry (helium) $[14,15]$. As for ND powders, manufacturers do not often provide this parameter. We estimated ND density (according to NIST, diamond density is $3.515 \mathrm{~g} / \mathrm{ml}$ ) assuming a linear dependence of nanofluid density on the volume fraction, which can be obtained if concentration (in $\mathrm{mg} / \mathrm{ml}$ ) is divided into $\rho_{n d}$ (constant); hence, slope $=1-\rho_{H_{2} O} / \rho_{n d}$. ND type does not dramatically change fluid density even for $50 \mathrm{mg} / \mathrm{ml}$ (Table 1). 
TABLE 1. Density study of aqueous dispersions of ND

\begin{tabular}{|c|c|c|c|c|}
\hline ND & Slope $\times 10^{4}$ & $R^{2}$ & $\rho_{n f l}, \mathrm{~g} / \mathrm{ml}$ (enhancement, \%) for $50 \mathrm{mg} / \mathrm{ml}$ & $\rho_{n d}, \mathrm{~g} / \mathrm{ml}$ \\
\hline RUDDM & $6.51 \pm 0.02$ & 0.99999 & $1.029 \pm 0.003(3.3)$ & $2.86 \pm 0.01$ \\
RDDM & $7.10 \pm 0.01$ & 0.99999 & $1.032 \pm 0.002(3.6)$ & $3.44 \pm 0.01$ \\
SDND & $7.21 \pm 0.03$ & 0.99997 & $1.033 \pm 0.003(3.6)$ & $3.57 \pm 0.01$ \\
\hline
\end{tabular}

TABLE 2. Thermal diffusivity and thermal conductivity of aqueous dispersions of ND

\begin{tabular}{|c|c|c|c|}
\hline Trademark & $\begin{array}{c}\text { Concentration, } \\
\mathrm{mg} / \mathrm{mL}\end{array}$ & $\begin{array}{c}\text { Thermal diffusivity } \times 10^{7}, \\
\mathrm{~m}^{2} \mathrm{~s}^{-1}\end{array}$ & $\begin{array}{c}\text { Thermal conductivity, } \\
\mathrm{W} \mathrm{m}^{-1} \mathrm{~K}^{-1} \text { (enhancement, \%) }\end{array}$ \\
\hline Water & - & $1.43 \pm 0.01$ & $0.595 \pm 0.008$ \\
\hline RUDDM & 0.2 & $1.48 \pm 0.01$ & $0.61 \pm 0.01(3)$ \\
& 1 & $1.49 \pm 0.01$ & $0.62 \pm 0.01(5)$ \\
& 4 & $1.53 \pm 0.01$ & $0.63 \pm 0.02(7)$ \\
\hline RDDM & 1 & $1.50 \pm 0.01$ & $0.61 \pm 0.02(4)$ \\
& 4 & $1.53 \pm 0.01$ & $0.63 \pm 0.02(7)$ \\
\hline SDND & 0.2 & $1.48 \pm 0.01$ & $0.61 \pm 0.01(3)$ \\
& 1 & $1.50 \pm 0.01$ & $0.62 \pm 0.01(5)$ \\
& 4 & $1.52 \pm 0.01$ & $0.63 \pm 0.02(7)$ \\
\hline
\end{tabular}

The specific heats vs. concentration dependences for the three ND fluids studied are presented in Fig. 1 (right). As seen from the Fig. 1, ND type does not influence the specific heat of the nanofluid significantly. The dependence on concentration $c(T=298 \mathrm{~K})$ is expressed by one and the same linear equation $C_{p, n f l}=(4.16 \pm 0.03)-(3.3 \pm 0.2) \cdot 10^{-3} c\left(R^{2}=0.98\right)$.

For specific heat of nanofluids, Pak and Cho [7] and Xuan and Roetzel [16] provided two expressions, $C_{p, n f}=\phi C_{p, n d}+(1-\phi) C_{p, f l}$ and $\left(\rho C_{p}\right)_{n f l}=\phi\left(\rho C_{p}\right)_{n d}+(1-\phi)\left(\rho C_{p}\right)_{f l}$. Model I and II, respectively, based on nanofluid volume fraction, $\phi$. DCS is the most commonly used technique for measuring specific heats [17]. Ghazvini et al. [18] presented a correlation of specific heat of nanofluids with a $1 \% \mathrm{w} / \mathrm{w}$ fraction of ND in engine oil as a function of temperature. Model I was used for the estimation of nanofluid specific heat for aqueous ND dispersions [9]. The results of our study do not correlate well with the theoretical equations of Models I or II. For Model I, poor correlation was observed in the whole concentration range, while Model II did work especially for highly concentrated dispersions. To make these calculations, the specific heat of NDs was measured $(0.75 \pm 0.04$ and $0.5 \pm 0.02 \mathrm{~J} / \mathrm{g} / \mathrm{K}$ for RUDDM and RDDM) and estimated values of ND density were used.

The experimental data on thermal diffusivity $\left(D_{T}\right)$ of the three ND fluids at different concentrations are given in Table 2. Plots for the thermal-lens signal on concentrations for all the three NDs are linear up to $0.2 \mathrm{mg} / \mathrm{mL}$ [3], at which point, they deviate due to changes in thermal conductivity. Thermal diffusivity was measured using the approach previously proposed for aqueous fullerene dispersions [2]. For concentrations below $0.1 \mathrm{mg} / \mathrm{mL}$ for all the NDs, it is negligibly different from water. For higher concentrations (Table 2), a $10 \%$ increase in thermal diffusivity is observed with good precision. Thermalconductivity was calculated as $k=\rho_{n f l} C_{p, n f l} D_{T}$ using the data and models for specific heat and density discussed above. The results show a 3 to $7 \%$ increase in thermal conductivity, which is in rather good agreement with the previous data [19-21]. Satisfactory precision of measurements should be specially mentioned. 


\section{Conclusions}

In this study, the appropriate precision of thermal lensing of aqueous nanodiamond dispersions was achieved, making possible accurate measurements of thermal diffusivity and thermal conductivity. A $3-7 \%$ increase in thermal conductive relative to pure water was confirmed. The TLS approach can be used for a more detailed characterization by the deconstruction of transient curves [2], which could provide the estimation of thermophysical properties of the dispersed phase, which would be useful for nanofluid characterization.

\section{Acknowledgements}

The work is supported by The Russian Science Foundation, grants no. 14-23-00012-P and 15-03-02168.

\section{References}

[1] Vargas H., Miranda L. Photothermal techniques applied to thermophysical properties measurements (plenary). Rev. Sci. Instrum., 2003, 74 (1), P. 794-799.

[2] Mikheev I.V., Usoltseva L.O., et al. Approach to the Assessment of Size-Dependent Thermal Properties of Disperse Solutions: TimeResolved Photothermal Lensing of Aqueous Pristine Fullerenes C60 and C70. J. Phys. Chem. C, 2016,120 (49), P. $28270-28287$.

[3] Volkov D.S., Semenyuk P.I., Korobov M.V., Proskurnin M.A. Quantification of nanodiamonds in aqueous solutions by spectrophotometry and thermal lens spectrometry. J. Anal. Chem. (Russ.), 2012, 67 (10), P. 842-850.

[4] Vul A.Y., Eydelman E., Inakuma M., Ōsawa E. Correlation between viscosity and absorption of electromagnetic waves in an aqueous UNCD suspension. Diam. Relat. Mater., 2007, 16 (12), P. 2023-2028.

[5] Sundar L.S., Sharma K., Naik M., Singh M.K. Empirical and theoretical correlations on viscosity of nanofluids: a review. Renew. Sustain. Energy Rev., 2013, 25, P. 670-686.

[6] Cheremisinoff N.P. Encyclopedia of Fluid Mechanics: Slurry Flow Technology. Gulf Publishing Company, Book Division, 1986.

[7] Pak B.C., Cho Y.I. Hydrodynamic and heat transfer study of dispersed fluids with submicron metallic oxide particles. Intern. J. Exp. Heat Transfer, 1998, 11 (2), P. 151-170.

[8] Vajjha R., Das D., Mahagaonkar B. Density measurement of different nanofluids and their comparison with theory. Pet. Sci. Technol., 2009, 27 (6), P. 612-624.

[9] Sundar L.S., Hortiguela M.J., Singh M.K., Sousa A.C. Thermal conductivity and viscosity of water based nanodiamond (ND) nanofluids: An experimental study. Intern. Commun. Heat and Mass Transfer, 2016, 76, P. 245-255.

[10] Yu Q., Kim Y.J., Ma H. Nanofluids with plasma treated diamond nanoparticles. Appl. Phys. Lett., 2008,92 (10), 103111.

[11] Sundar L.S., Singh M.K., Sousa A.C. Enhanced thermal properties of nanodiamond nanofluids. Chem. Phys. Lett., 2016, 644, P. 99-110.

[12] Taha-Tijerina J.J., Narayanan T.N., et al. Nanodiamond-Based Thermal Fluids. ACS Appl. Mater. \& Interfaces, 2014, 6 (7), P. 4778-4785.

[13] Kidalov S., Shakhov F., Vul A.Y. Thermal conductivity of nanocomposites based on diamonds and nanodiamonds. Diam. Relat. Mater., 2007, 16 (12),P. 2063-2066.

[14] Larionova I., Frolov A., Poleva L., Bychin N. Study of the composition and physicochemical properties of diamond hydrogels. Colloid J., 2004, 66 (3), P. 372-374.

[15] Larionova I., Kuznetsov V., et al. Properties of individual fractions of detonation nanodiamond. Diam. Relat. Mater., 2006, 15 (11), P. $1804-1808$

[16] Xuan Y., Roetzel W. Conceptions for heat transfer correlation of nanofluids. Intern. J. Heat and Mass Transfer, 2000, 43 (19), P. $3701-$ 3707.

[17] Shahrul I., Mahbubul I., Khaleduzzaman S., Saidur R., Sabri M. A comparative review on the specific heat of nanofluids for energy perspective. Renew. Sustain. Energy Rev., 2014, 38, P. 88-98.

[18] Ghazvini M., Akhavan-Behabadi M., Rasouli E., Raisee M. Heat transfer properties of nanodiamondengine oil nanofluid in laminar flow. Heat Transfer Engin., 2012, 33 (6), P. 525-532.

[19] Yeganeh M., Shahtahmasebi N., et al. Volume fraction and temperature variations of the effective thermal conductivity of nanodiamond fluids in deionized water. Intern. J. Heat and Mass Transfer, 2010, 53 (15), P. 3186-3192.

[20] Torii S. Experimental study on thermal transport phenomenon of nanofluids as working fluid in heat exchanger. Intern. J. of AirConditioning and Refrigeration, 2014, 22 (01), 1450005.

[21] Jang S.P., Choi S.U. Cooling performance of a microchannel heat sink with nanofluids. Appl. Therm. Eng., 2006, 26 (17), P. $2457-2463$. 